\title{
Simulation of mining-induced seismicity using the Salamon-Linkov method
}

\author{
D Malovichko Institute of Mine Seismology, Australia \\ G Basson Institute of Mine Seismology, Australia
}

\begin{abstract}
An approach to model seismicity induced by mining was proposed by MDG Salamon and developed further by AM Linkov. We suggest a variation of the Salamon-Linkov method that does not require a priori assumptions about sizes, orientations and locations of flaws, reducing the amount of input data. The algorithm places a 3D grid over the model domain and searches the entire grid space for possible planes of failure by identifying lobes of positive Coulomb Failure Stress (CFS). The largest CFS lobes are then utilised as flaws. Another change from the original formulation is that the flaws are discretised into smaller sub flaws. This accounts for the complexity of the shapes of the flaws and provides more accurate modelling of their effect on the stress field. Some elements of the original framework of Salamon and Linkov, e.g. tensile failure and creep effects are not included into our implementation. This is done intentionally to keep the method simple at the current stage.
\end{abstract}

The implemented method requires limited input data: models of mining steps, information about virgin stress, rock mass elastic properties and strength. The strength may be anisotropic and inhomogeneous, where specific Mohr-Coulomb failure criteria can be assigned to particular orientations (describing joint sets) and 3D surfaces (describing faults or dykes). The output of the method represents two catalogues - one for seismic and another for aseismic events. The catalogues contain origin times (mining steps), coordinates, seismic potencies and source mechanisms of events.

The method was applied to real case studies at two mines. The Boundary Element Method (BEM) models of the mines were of the order of 200,000 Fictitious Force (FF) elements. The first model included seven mining steps and the second model consisted of 33 steps. The computations took seven and 50 hours respectively on a multi-core machine. In the first case the resulting catalogue includes more than 900 seismic events, whereas in the second case the catalogues contained approximately 3,400 seismic and 100 aseismic events.

The modelled seismicity may be used as a reference for the interpretation of the observed seismicity. Analysing the discrepancies between the modelled and observed seismicity can also help to assess the validity of input parameters - characteristics of in situ stress and failure criteria. Another possible application of the suggested method is the testing of future mining scenarios. Although the prediction of seismic response for individual scenarios may be not accurate, the difference between the modelled seismicity of the scenarios may help to choose the optimal one in regards to seismic or ground motion hazard.

\section{Introduction}

In his pioneering work Salamon (1993) suggested a simple computational scheme that makes it possible to simulate seismicity associated with mining. The ideas of Salamon were extensively elaborated and improved by Linkov. His computational framework allows to model both seismic and aseismic events, reproduce creep effects of the induced and triggered seismicity. The details of the framework are presented in Linkov $(2005,2013)$. The similar aspects of modelling of seismicity associated with mining were explored by other researchers (Sellers \& Napier 2001; Spottiswoode 2001). 
In this paper we suggest a variation to the method, which reduces the amount of input data. Our implementation follows the framework of Linkov. However some parts of it, e.g. modelling of creep effects, accounting for tensile failure, were intentionally left outside the scope of work.

Various aspects of the implemented method are discussed in the first section of the paper. The method was applied to real case studies of actual mining sequences at two mines. The results are presented in the second section of the paper.

\section{$2 \quad$ Method}

The main idea of the Salamon-Linkov method is to identify the possible failure of flaws in the elastic rock mass model and then to transfer the parameters of modelled failure into a seismological domain. As mentioned in (Linkov 2013), the method may utilise an arbitrary numerical code that solves an elastostatic problem (BEM, FEM, FDM, DEM and the like). Our implementation is based on the Indirect Boundary Element Method. Figure 1 highlights the differences between the original method of Salamon, improved method of Linkov and our implementation. These are as follows:

- The methods of Salamon and Linkov imply that the modelled domain is initially preseeded with flaws with the prescribed spatial, orientation and size distributions (variants (a) and (b) in Figure 1). We have tested an alternative approach whereby an algorithm tries to find the optimal failure planes within the rock mass. This means no a priori assumptions about sizes, orientations and locations of flaws are made.

- In the method of Salamon only mining induced stress is used to check the fulfilment of failure criterion for the flaws, while in the version of Linkov the effect of other flaws on stress field is also taken into account.

- Salamon and Linkov monitor and control the behaviour of preseeded weaknesses according to stress at their centroids. In our approach all flaws are discretised and slips of patches on the flaws are evaluated accounting not only mining induced stress, but also the effect of other patches and flaws as well.

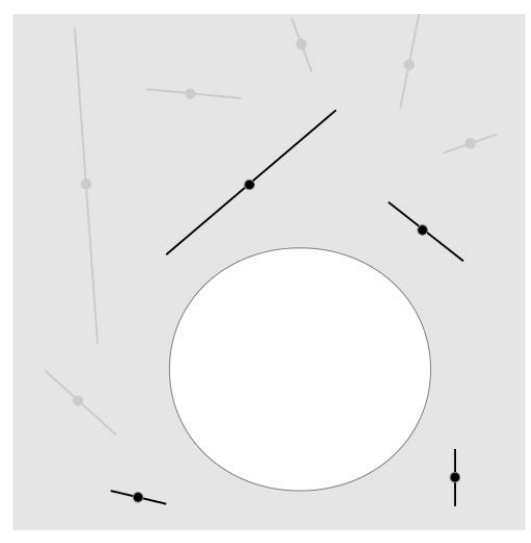

(a)

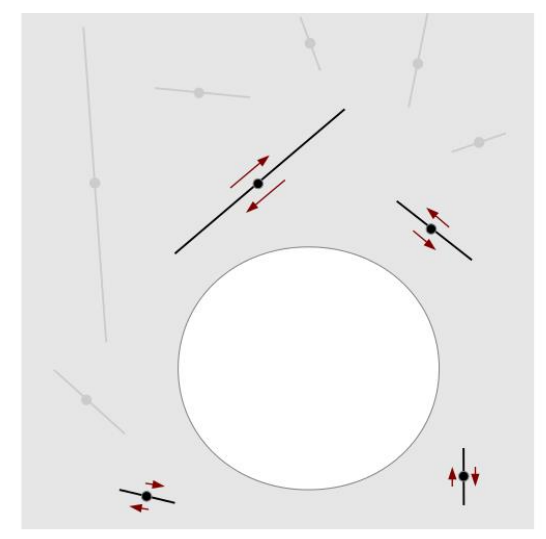

(b)

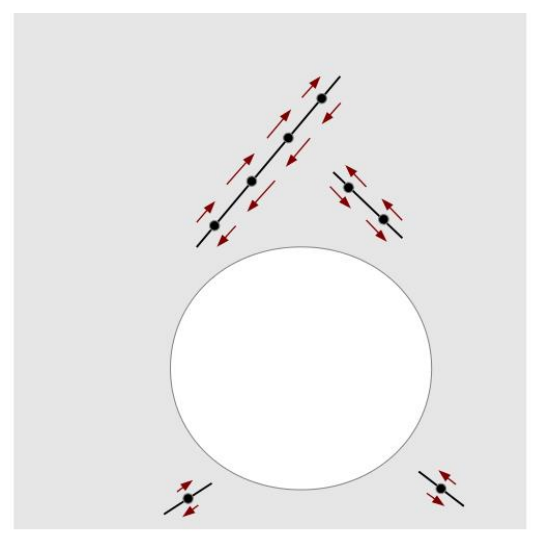

(c)

Figure 1 Illustration of the differences between the original technique of Salamon (a); development of Linkov (b); and our implementation (c). The disturbance of stress in modelled domain (grey rectangle) by the mining (white circle) results in slips along the flaws (black lines with dots)

Figure 2 provides an overview of the implemented method. 


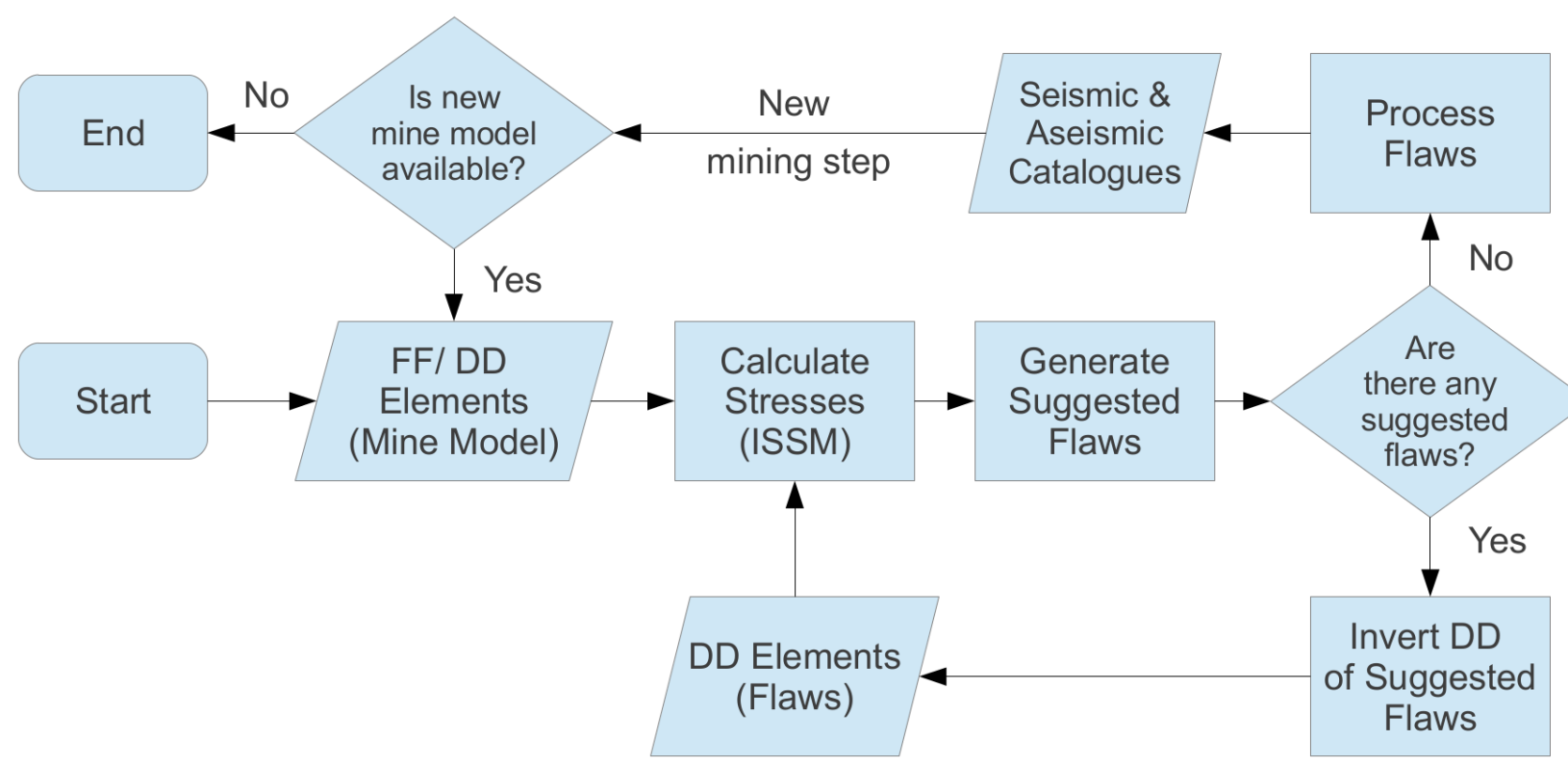

Figure 2 Flow chart of the implemented variation of the Salamon-Linkov method

\subsection{Algorithm}

Initially the numerical models (consisting of either displacement discontinuity (DD) or FF elements) of all the consecutive mining steps involved during the simulation are build and solved separately using the in-house Indirect Boundary Element Software ISSM. These solutions are stored for later use.

The numerical model of the first mining step is identified and used to start the process. A bounding grid is placed around the area of interest within the numerical model and a stress tensor is obtained at each grid point from the solution of the model. The stress field contains information about the virgin stress of the simulated rock mass as well as the influence of the model. A flaw generating algorithm (also developed inhouse), then uses the calculated stress field to identify possible planes or surfaces of failure inside the domain. For future reference these planes and surfaces are called 'suggested flaws'. The flaw generating algorithm will be discussed in more detail in Section 2.3.

The suggested flaws are discretised into a set of DD elements, and the magnitudes of these elements are evaluated using relations provided in (Linkov 2013). These DD elements, together with the elements of the numerical model are then supplied to the ISSM code to calculate stresses. After that Flaw Generating algorithm again identifies new possible planes of failure. This process is repeated until the algorithm produces no new flaws. This means that the planes or surfaces, which meet the failure criteria, cannot be found within the volume of interest with selected spatial discretisation. At that stage the flaws accumulated on the current mining step are compared with the flaws accumulated on the previous step. The difference between these represent either newly created flaws or reactivated flaws. Both are ported to the catalogues of seismic and aseismic events with the time mark corresponding to the current mining step. The DD elements of the accumulated flaws are then used together with the solution of the next mining step numerical model to once again calculate stress fields on the bounding grid as described above.

The above mentioned process is repeated until all the mining steps are evaluated.

\section{$2.2 \quad$ Input data}

The implemented method requires the following information:

- FF or DD models of the mining steps.

- In situ stress and elastic rock mass parameters.

- Shear failure criteria. 
The shear strength of flaws is described by the Mohr-Coulomb criterion, which is parameterised by peak cohesion $c_{p}$, peak friction angle $\varphi_{p}$, residual cohesion $c_{r}$ and residual friction angle $\varphi_{r}$. The simplest option is to assume a single Mohr-Coulomb failure criterion for the entire rock mass. However the spatial variability and anisotropy of the strength can also be taken into account in our implementation. For example joint sets with particular orientations (described by dip and dip direction) may have different failure properties as compared to the surrounding rock mass. The specific failure criterion may also be prescribed to particular 3D surfaces, e.g. weak faults and dykes.

The discrimination between stable and unstable behaviour of flaws is governed by a softening modulus $M_{C}$ that is assigned independently to rock mass, joint sets and 3D surfaces (faults). This allows for flexibility when modelling the seismic and aseismic response. For example, specifying a high softening modulus for the rock mass and low modulus for 3D fault surface results in brittle failure within the rock mass (seismic events) and stable slip along the fault (aseismic events).

The implemented variation of the Salamon-Linkov method also requires setting of the following:

- Volume of interest (rectangular block) which forms the bounding grid as described in Section 2.1. This box can cover the entire model or it focus on a small area of the domain.

- Parameters of spatial gridding - spatial step $\Delta x$ and angular step $\Delta \alpha$. The spatial step is used to discretise the flaws. Both $\Delta x$ and $\Delta \alpha$ are also utilised in the Flaw Generating algorithm (described below). Effectively the spatial step specifies the size of smallest seismic and aseismic events.

\subsection{Generation of suggested flaws}

The following computational scheme is used to derive the potential (suggested) flaws from the discretised stress field described in Section 2.1:

- The volume of interest is considered.

- The volume is intersected by planes of various orientation (with dip and dip direction step $\Delta \alpha$ deg.) spaced by $\Delta x \mathrm{~m}$ from each other. These planes and fault plane surfaces within the volume of interest are discretised using square elements of size $\Delta x$.

- For each plane or surface:

- The distribution of Coulomb Failure Stress is calculated: $C F S=\tau-\left[c_{p}-c_{r}+\sigma_{n}\left(\tan \varphi_{p}-\tan \varphi_{r}\right)\right]$, where $\tau$ is the shear stress along the element of plane or surface and $\sigma_{n}$ is the normal confining stress. The parameters of Mohr-Coulomb criterion $c_{p}, \varphi_{p}, c_{r}$ and $\varphi_{r}$ of the considered element may correspond to the failure criterion of either the rock mass, or joint set(s), or 3D surfaces fault(s) depending on location and orientation of the element.

- The isolated lobes of positive CFS which fit the model of elliptic crack are identified. These are expressed as seismic sources with prescribed coordinates and sizes. The events form the catalogue of events.

- The resulting catalogue of seismic events is cleaned. This means that all sources that are located within the source radius of a larger source are removed.

- Finally, the planes and surfaces corresponding to the events in the cleaned catalogue are considered as suggested flaws.

The phases of the method described above are illustrated in Figure 3. 

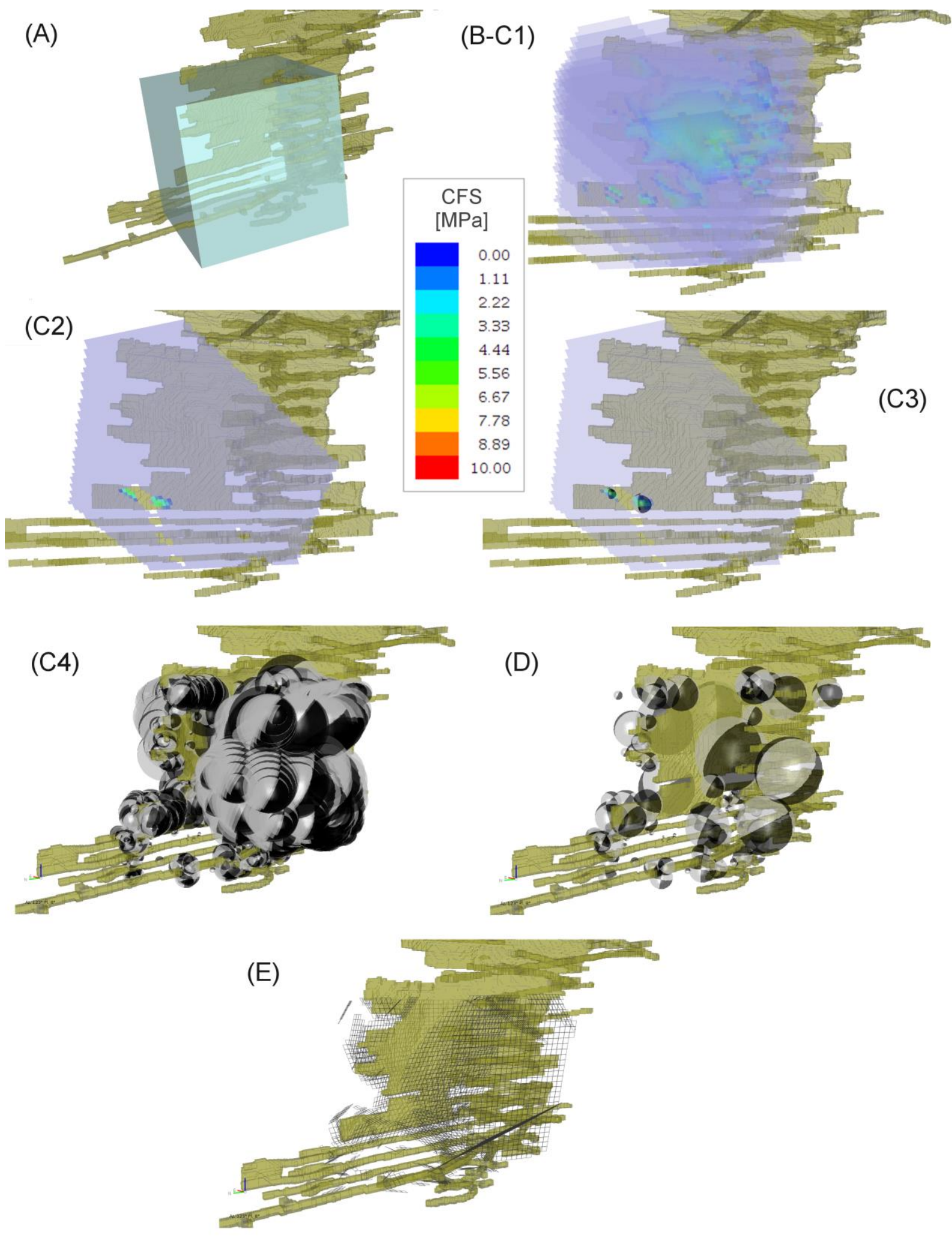

Figure 3 Illustration of the procedure of generation of suggested flaws. Region of interest (A); distribution of Coulomb Failure Stress (CFS) on a set of parallel planes of a fixed orientation (B-C1); example of the distribution of CFS on a plane intersecting the orebody (C2); the plane contains two patches of positive CFS, which can be described as seismic events (C3); primary set of events composed from isometric CFS lobes of all tested planes (C4); and cleaned set of events (D); the discretised flaws that correspond to the cleaned set of events (E) 
Figure 3(B) shows that CFS lobes on the adjacent planes have the same genesis and the release of shear stress on one of these planes should result in elimination of CFS on the neighbouring planes. Thus it is not efficient to consider all CFS lobes as suggested flaws. That is why we try to eliminate the redundant flaws by manipulating the catalogue of equivalent seismic events. The primary catalogue of seismic events composed from all CFS lobes (e.g. shown in Figure 3(C4)) will contain the duplicates. The elimination of events, which are located within the source radius of a larger event, reduces the number of events and provides a cleaned catalogue (e.g. shown in Figure 3(D)). The discretised planes and surfaces corresponding to the cleaned catalogue of events $(E)$ are transferred to the next step of the method (Figure 2) - inversion of DD of suggested flaws.

\subsection{Output}

The method provides two types of output information:

- Mechanical: (A) sets of DD elements describing total accumulated flaws for each mining step, and (B) sets of DD elements characterising the newly created and reactivated flaws at each mining step.

- Seismological: catalogue of seismic events and catalogue of aseismic events. Each catalogue contains origin time (equivalent to mining step), source coordinates, scalar potency, source mechanisms and size.

Salamon and Linkov suggest a way to derive the seismic energy of events from mechanical parameters of flaws (Salamon 1993; Linkov 2013), however currently this is not used in our implementation of their method. Our catalogues of modelled events do not contain seismic energy since the derived seismic energy is less accurate as compared to the static source parameters (seismic potency and source mechanism). At the early stage of testing and application of the method it makes sense to limit the analysis to the source parameters of a better quality.

The obtained catalogue of modelled events may be examined in the same way as actual seismic data. Conventional seismological data analysis software makes it possible to filter the modelled events in space, time and parameter domains, as well as analyse time history and size distribution of events, stereonet of mechanisms.

\section{$3 \quad$ Case studies}

\subsection{Barrick, Hemlo Operations: Williams Mine, Canada}

Our algorithm was applied to a real mining sequence case study in the mine, which utilises alimak and longhole open-stoping methods.

The required input information (virgin stress, shapes and sequence of mined-out volumes, strength characteristics of rock mass) was provided by the mine. In this case we did not consider the inhomogeneities and anisotropy of strength characteristics. A single Mohr-Coulomb failure criterion was used for the entire rock mass. The BEM model representing the excavations consisted of approximately $197,000 \mathrm{FF}$ elements for the first mining step and 207,000 elements for the final step.

In total it took seven hours to run the method over all seven mining steps. More than 7,000 DD elements describing shear failure in the model were accumulated during the simulation. These correspond to about 4,000 seismic events. The first 3,100 events simulated for the initial mining model can be disregarded as they effectively describe response to instantaneous mining of all excavations existing before the first mining step. The seismic response to the subsequent seven mining steps comprises 900 events.

Figure 4 illustrates the details of the calculations - accumulated flaws for the 3rd mining step (September 2013), as well as new and reactivated flaws associated with the 4th mining step (October 2013). 
The seismological output of the method is shown in Figure 5 along with the actual recorded seismicity for the corresponding time period.

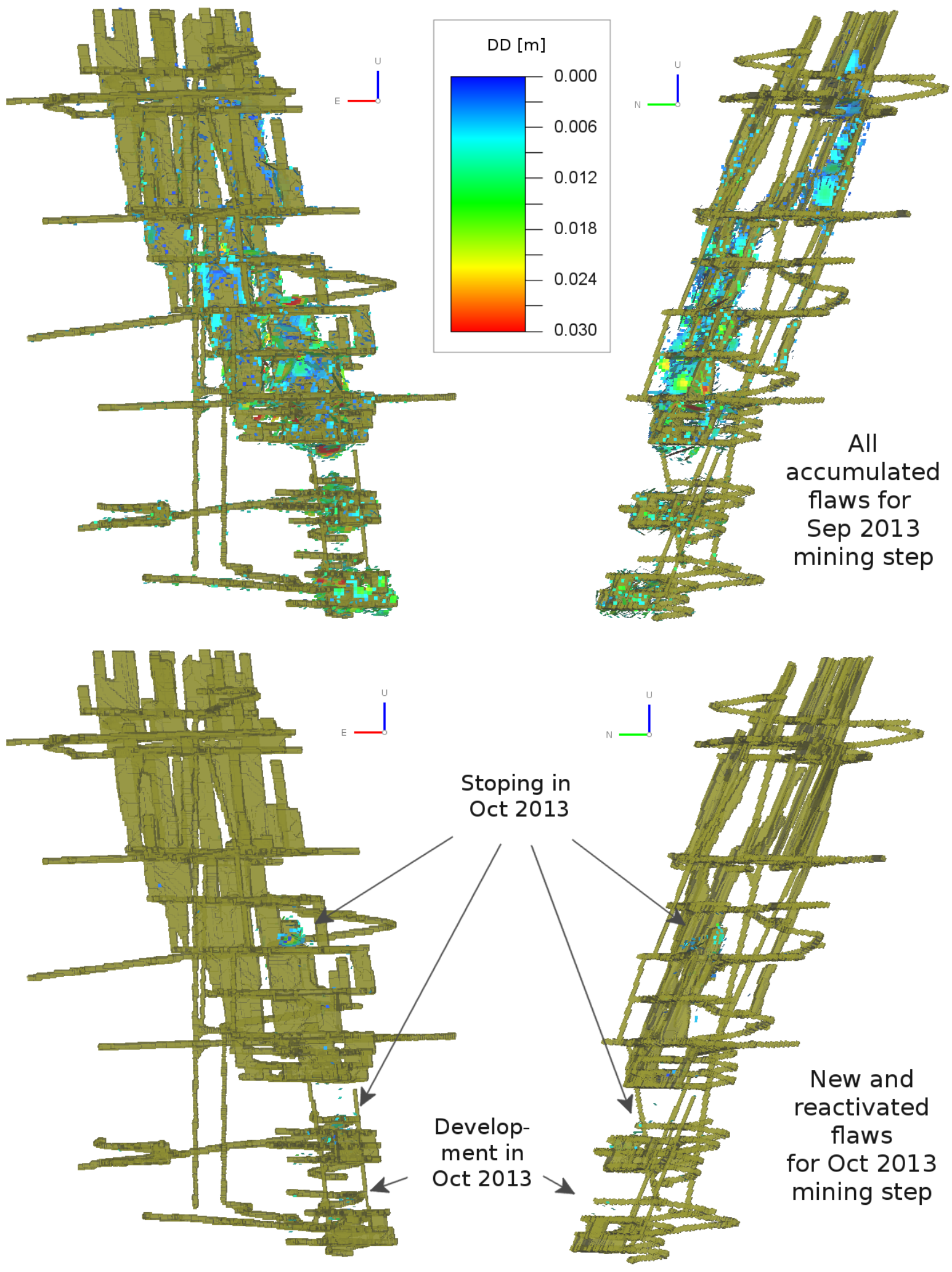

Figure 4 Illustration of the application of Salamon-Linkov method to a case. Top:

Fictitious Forces model of excavations for September 2013 (yellow wire-frame) and DD elements describing the accumulated shear failures (coloured meshes). Bottom: the new and re-activated DD elements associated with October 2013 mining step 

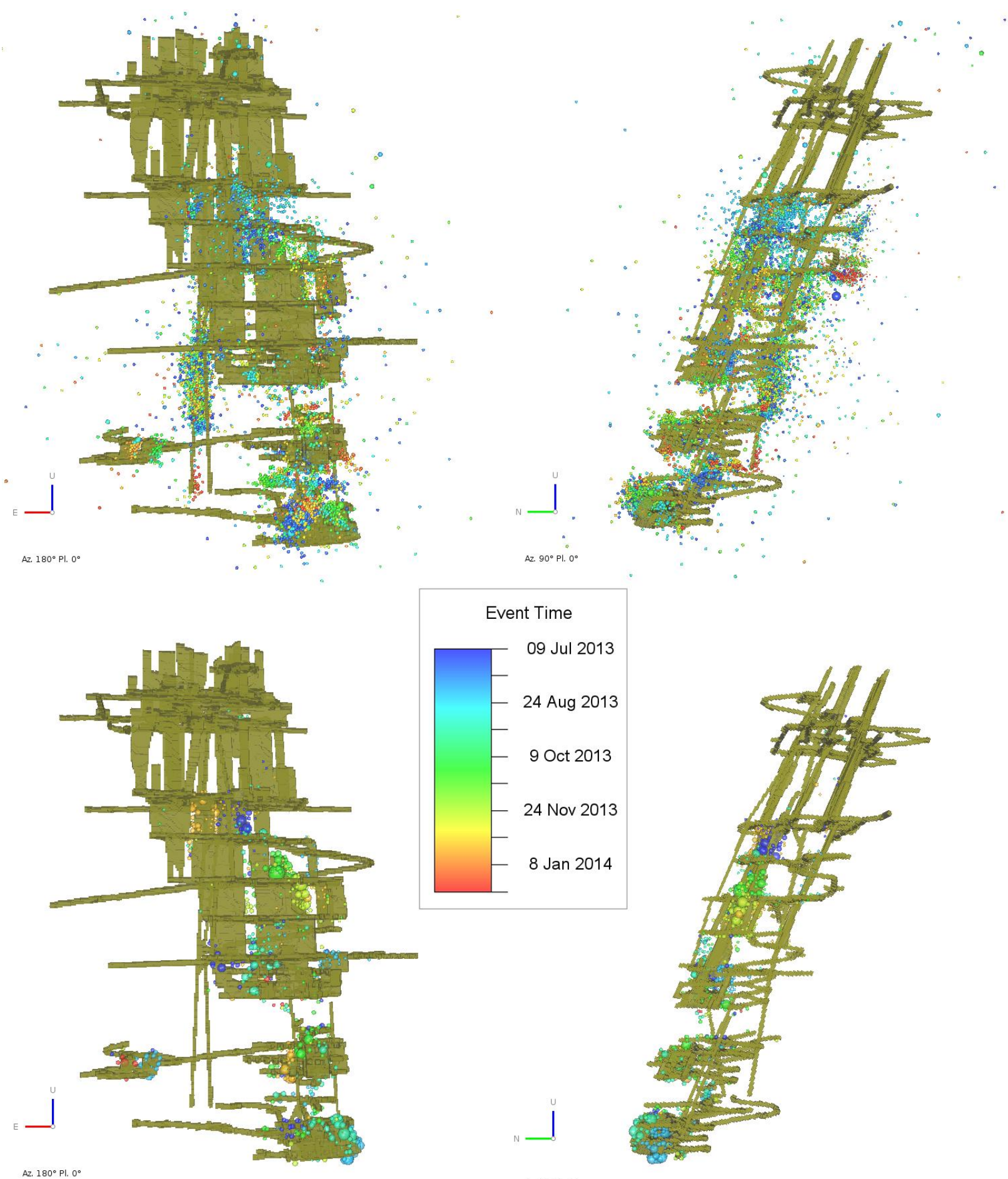

Figure 5 Observed (top) and modelled (bottom) seismicity at Williams Mine for the period July 2013 - January 2014. Monthly mining steps were used in the modelling. Events are coloured according to time and sized according to $\log P$. Common colouring and size scale are used for the observed and modelled events

The comparison of modelled and observed seismicity is outside the scope of this paper. However, for demonstration purposes, in Figure 6 we show the differences in the mechanisms of recorded and modelled seismic events for a particular area of the mine. Events from both datasets correspond to reverse and 
strike-slip faulting. For the real data the P-axis of mechanisms is generally oriented along the strike of the orebody, while for the modelled dataset it has opposite direction.

Observed seismicity
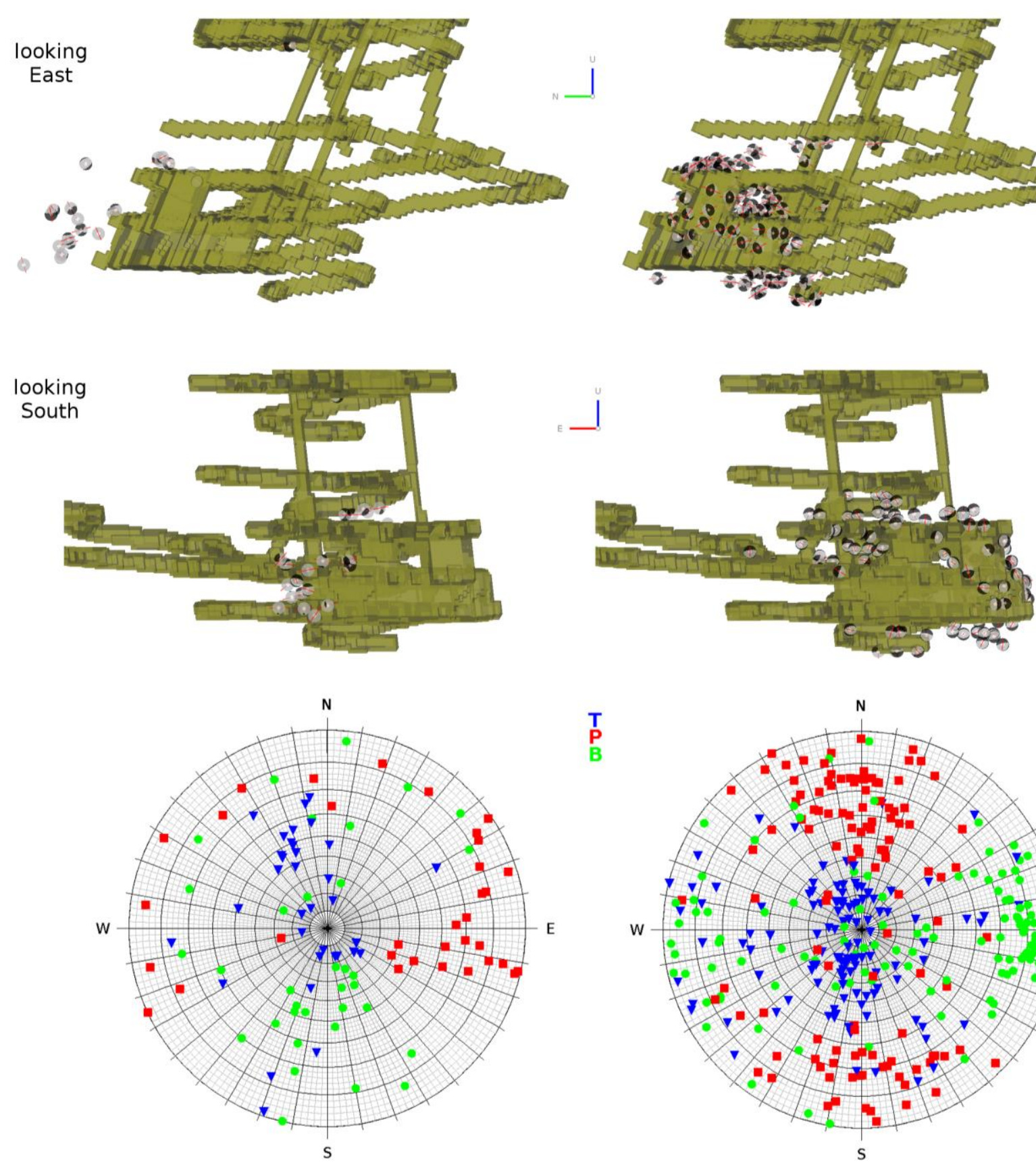

$\mathrm{T}$
$\mathrm{P}$
$\mathrm{B}$

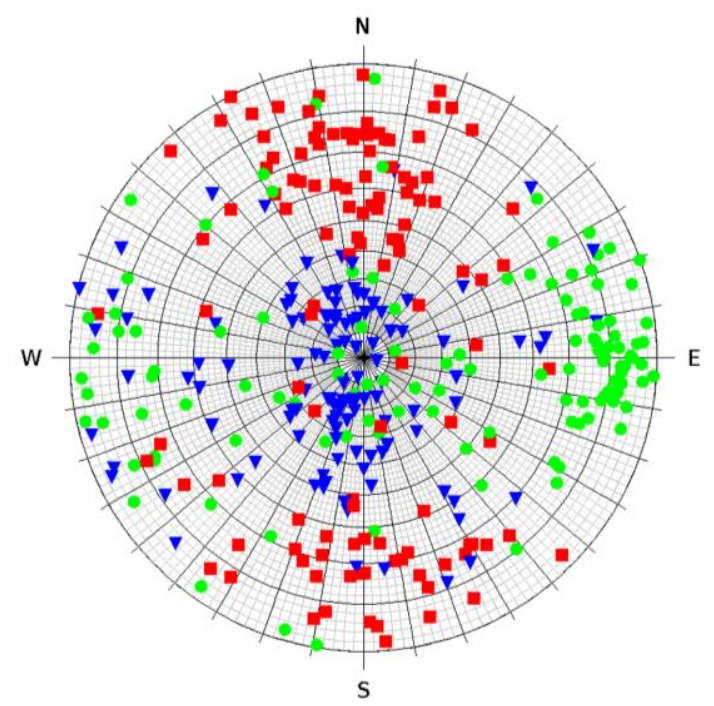

Figure 6 Comparison of seismic source mechanisms of the recorded (left) and modelled (right) events for the deep part of the mine. All modelled events are shown in the plot and stereonet, while only a limited subset of data is used for the observed seismicity

\subsection{Renison Mine, Australia}

Another application case study was carried out for the Lower Federal narrow vein mining area of Renison Mine. 
The seismic system at the mine has a number of triaxial geophones installed in the solid rock mass away from the stopes and decline. This provides high quality seismic data; waveforms of events with clear arrivals of $\mathrm{P}$ - and $\mathrm{S}$-waves. As a result the location, source parameters and source mechanisms of events are reliable.

The recorded seismicity has a number of characteristic features:

- There is a long-range correlation between the strong disturbances, e.g. mining of closing pillars, larger events, and seismic response. For example seismic events followed by the firing of a closing pillar are not clustered just around the pillar, but also spread over the distance of up to 100-120 m.

- When analysing the mechanisms of the seismic events it shows that a particular joint set (dip 50', dip direction $130^{\circ}$ ) plays an important role in the seismicity.

- Seismic response in the southern part of the mine is lower as compared to the northern part. Qualitatively it may be explained by a presence of weak dyke in the southern part.

We tried to reproduce these features in the modelled seismicity.

The BEM models of 33 monthly mining steps for the period from July 2011 - March 2014 were built. The first model consisted of approximately 143,000 FF elements, and the final model of 183,000 elements.

Initially we tried to use a single failure criterion for the entire rock mass. The peak cohesion $c_{p}$ and peak friction angle $\varphi_{p}$ were recommended by the geotechnical engineer of the mine. However these parameters resulted in the modelled seismicity being clustered too close to the excavations. The cohesion and friction angle of the rock mass were lowered to simulate a larger scattering of seismicity around the orebody (Table 1). Also specific failure criteria of a joint set (dip $50^{\circ}$, dip direction $130^{\circ}$ ) and weak dyke in the southern part of the mine were prescribed. The softening modulus $M_{C}$ of the dyke was set to zero to model stable slip along it. For the rock mass and the joint set $M_{C}$ was set high to simulate brittle failure.

\section{Table 1 Strength characteristics}

\begin{tabular}{cccccc}
\hline & $\begin{array}{c}\text { Peak cohesion } \\
c_{p} \text { (MPa) }\end{array}$ & $\begin{array}{c}\text { Peak friction } \\
\text { angle } \varphi_{p} \\
\text { (deg.) }\end{array}$ & $\begin{array}{c}\text { Residual } \\
\text { cohesion } c_{r} \\
\text { (MPa) }\end{array}$ & $\begin{array}{c}\text { Residual } \\
\text { friction angle } \\
\varphi_{r} \text { (deg.) }\end{array}$ & $\begin{array}{c}\text { Shear softening } \\
\text { modulus } M_{C} \\
\text { (GPa) }\end{array}$ \\
\hline Rock mass & 5 & 35 & 0 & 35 & 100 \\
Joint set & 4 & 27 & 0 & 27 & 100 \\
Dyke & 2 & 12 & 0 & 12 & 0 \\
\hline
\end{tabular}

It took approximately 50 hours to run the algorithm over all 33 mining steps. More than 14,200 DD elements describing flaws in the model were accumulated during the simulation. These corresponded to about 4,900 seismic events. The first 1,500 events can be disregarded as they effectively describe response to the instantaneous mining of all excavations existing before July 2011. The response to the subsequent 33 mining steps comprises 3,400 seismic and 100 aseismic events.

Figure 7 illustrates the details of the calculations for a 27th mining step (September 2013).

As it is expected the aseismic events are distributed along the weak dyke. The location of modelled seismic events is shown in Figure 8 along with the recorded seismicity. The modelled seismicity mimics the scattering of events in the northern part of the domain; however in the south the synthetic seismicity is still greater as compared to the observed seismicity. This means that the incorporation of weak dyke does not allow to release enough of the stress in the southern part of the mine aseismically. 
Comparing the sizes of seismic events (expressed in terms of $\log P$ ) shows a general agreement for large events. The modelled seismicity has much more middle-size and much less small-size events as compared to the observed seismicity. This reflects the limitation on the minimum size of flaws, which was set to $5 \mathrm{~m}$.
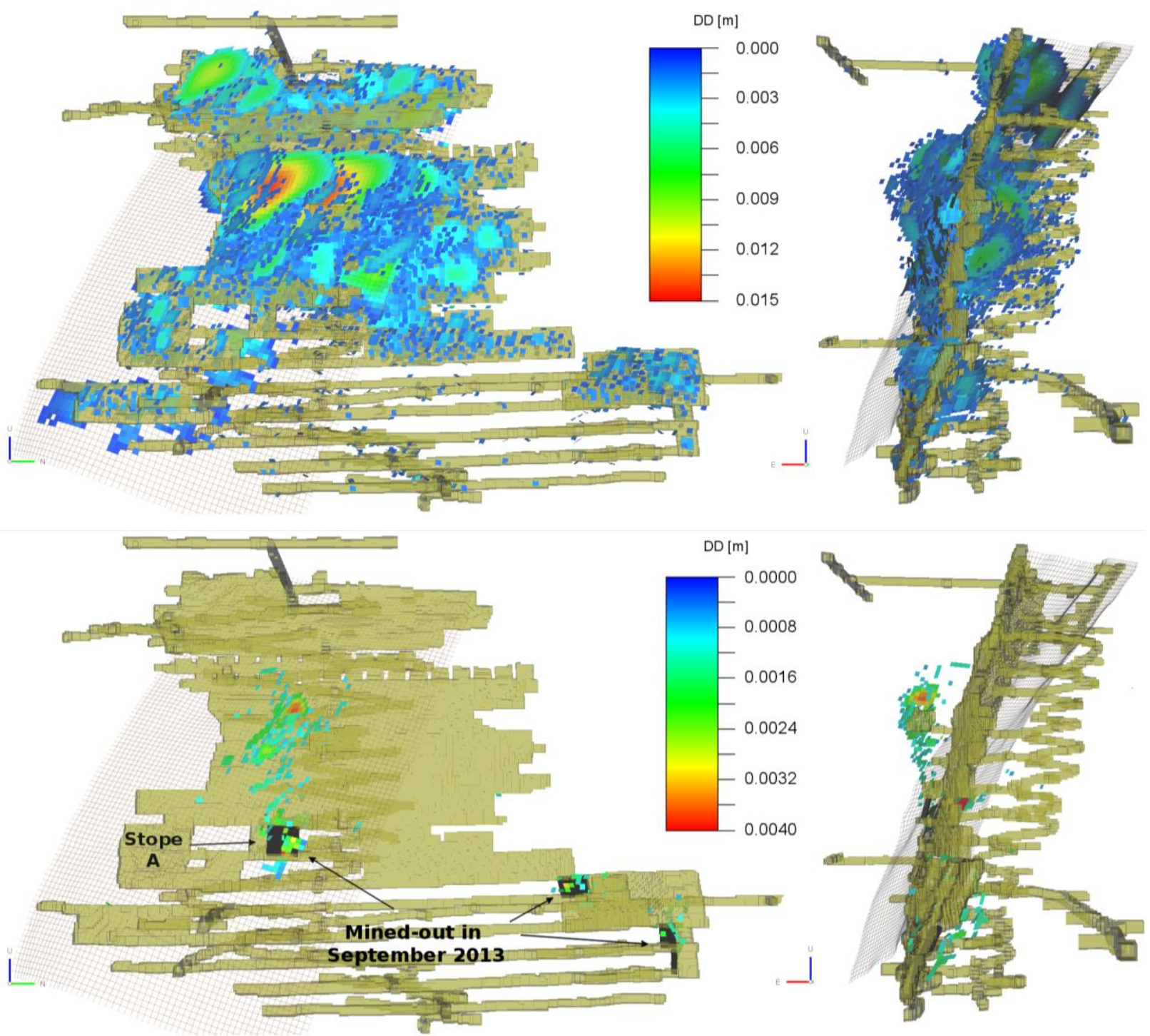

Figure 7 Illustration of the application of the Salamon-Linkov method for mining sequence at Renison Mine. The FF model of excavations for August 2013 is shown using yellow solid wire-frame and the weak dyke is displayed as a brown mesh. Top: the DD elements describing the accumulated flaws (coloured meshes). Bottom: the new and re-activated DD elements associated with September 2013 mining step. The stopes mined-out in September 2013 are marked in black. Each DD element characterises either seismic or aseismic events. Note a long-range correlation between the mining and seismic response in the central part of the mine - the extraction of stope A initiated slip along the flaws $\mathbf{8 0 - 1 3 0 ~} \mathrm{m}$ higher in the hanging wall

\section{Discussion and conclusions}

The application of the implemented method to real case studies highlighted several advantages. They are:

- Reasonable computational time. 
- Limited amount of input data. The geometry of mined out voids, elastic properties of the rock mass and parameters of virgin stress are generally available at the mines. The strength may be described by Mohr-Coulomb failure criterion. The above mentioned is enough to run the modelling of seismicity using the suggested method. The original formulation of the method by Salamon and Linkov requires extra input information - the distributions describing location, orientation and size of flaws.

- Flexibility in modelling of seismic and aseismic response. The decision regarding qualification of a particular flaw as seismic or aseismic may be done retrospectively, i.e. after the modelling of seismicity for all mining steps is completed.

- Mimicking a number of features of induced and triggered seismicity. One of these is the remote triggering of seismic events discussed in a number of papers (McKinnon 2006). The size distributions of modelled seismic events share similarities with a power law distribution. In our implementation it is not predefined by the input data, as the flaws are derived from the stress model.

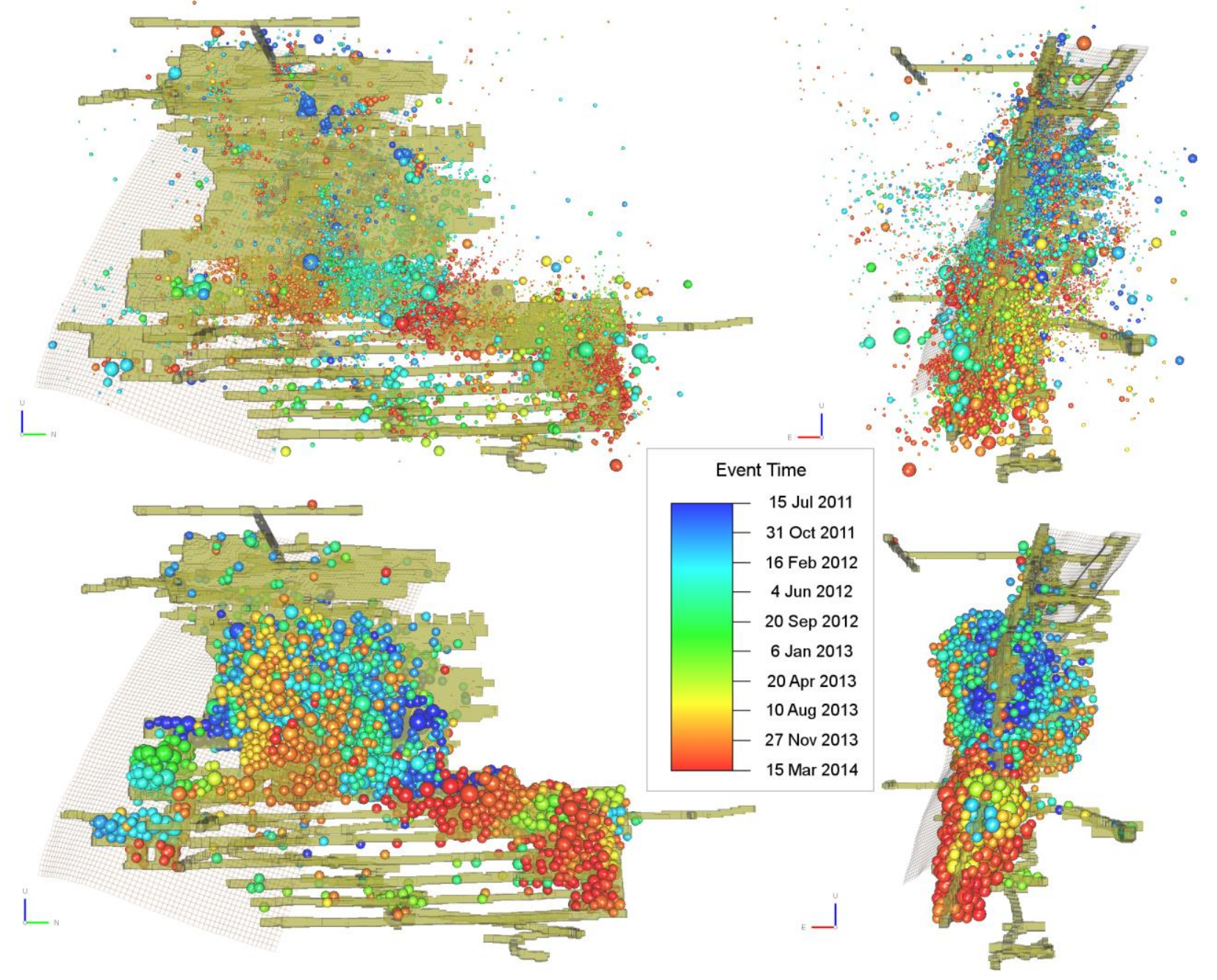

Figure 8 Seismicity recorded in the Lower Federal area of Renison Mine for the period July 2011 - March 2014 (top). Modelled seismicity associated with 33 corresponding monthly mining steps (bottom). Events are sized according to $\log P$

The major disadvantages of the method are: 
- Approximate modelling of the stress field. The BEM models of mining steps are solved in advance and are not affected by the accumulating flaws. A rigorous implementation would be to solve the model of excavations along with the DD elements describing the flaws at each iteration (Figure 2). However, this will increase the computational costs of the method. The accuracy of this approximation is a topic of future study.

- Neglect of tensile failure. The original method of Salamon and Linkov considers the tensile mode of failure of flaws and utilises a conventional description of tensile seismic sources. The latter presumes that a tensile crack occurs in a homogeneous medium and therefore the corresponding source mechanism has significant explosive component (strong P-wave with positive polarities Figure 9(a)). When a tensile crack happens in the vicinity of excavation (stress fracturing/ dog-earing) and the source size is of the order of the excavation dimension, then the source mechanism should rather contain an implosive, than explosive component. This is due to the fact that the source is driven by compression in the direction of local maximum principal stress, then dilatation normal to the plane (Figure 9(b)). Elastic convergence of the excavation also adds an implosive component to the seismic source mechanism. In future we are planning to incorporate tensile flaws in the modelling method, however representation of the corresponding seismic source will be different from the conventional one.

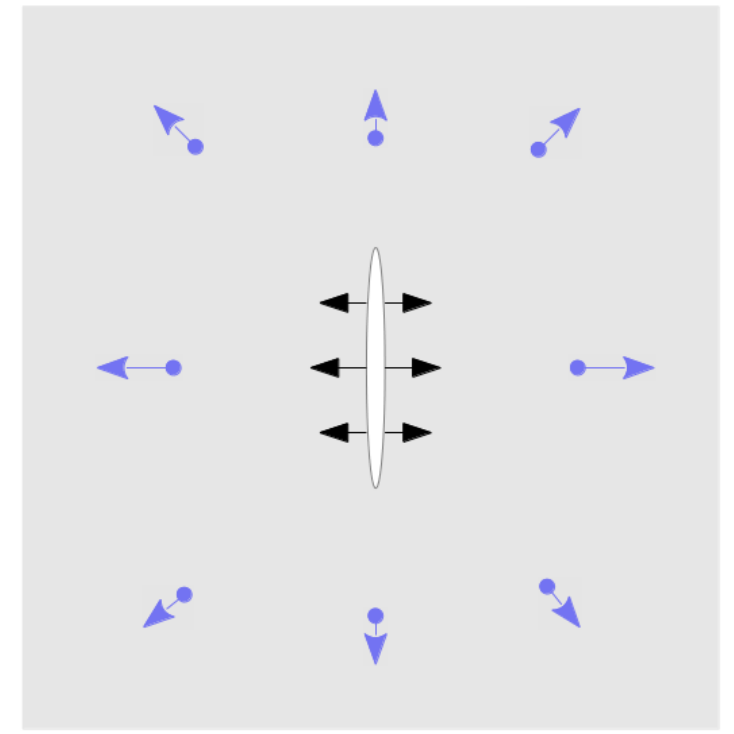

(a)

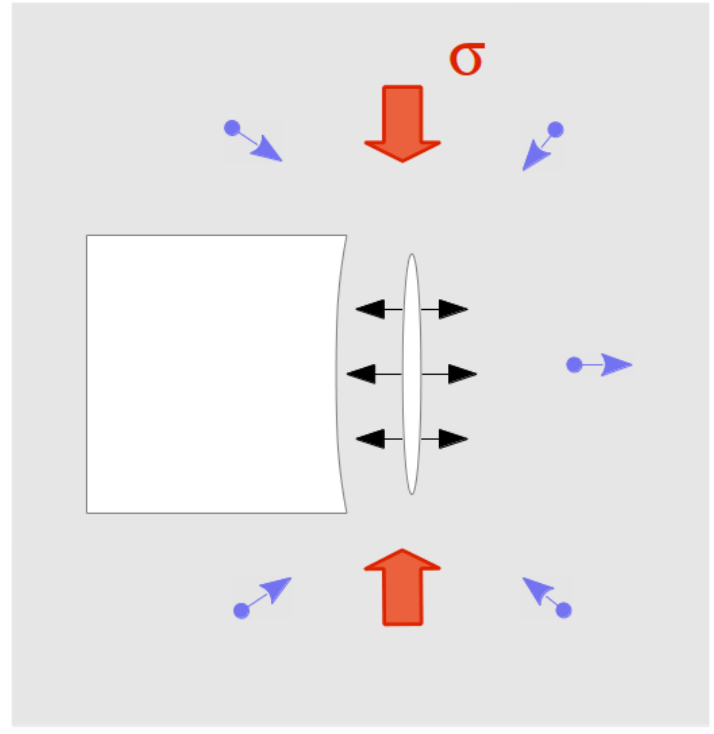

(b)

\section{Figure 9 Seismic radiation from a tensile crack in an unconfined medium (a); and in the vicinity of the excavation (b)}

The observed and modelled seismicity may be compared in numerous ways. Arbitrary parameters of seismicity can be correlated between observed and modelled catalogues of seismic events both in time and spatial domains. However some parameters are not suitable for this task. For instance, the number of seismic events or rate of seismic activity are no good candidates because they do not take into account the size of events. Furthermore the rate of observed seismicity may also be sensitive to the performance of seismic system. Seismic energy is also not an optimal candidate as its derivation for synthetic events is more model-dependent as compared the static source parameter - seismic potency. The accuracy of seismic energy in the catalogue of observed events is also generally lower than for the seismic potency. We think that the following two source characteristics provide an optimal way of comparing the observed seismicity with the seismicity modelled by the suggested method:

- Seismic potency, which measures the amount of coseismic deformation.

- Source mechanism, which describe the geometry of coseismic deformation. 
The implemented variant of Salamon-Linkov method does not take into account uncertainty of the input data, e.g. failure criteria. Thus it is unlikely that it can be used for accurate prediction of seismicity. The modelled seismicity may rather be used as a reference for the interpretation of the observed seismicity. Analysing the discrepancies between the modelled and observed seismicity can also help to assess the validity of input parameters - characteristics of in situ stress and failure criteria.

Another possible application of the method is the testing of future mining scenarios. Although the prediction of seismic response for individual scenarios may be not accurate, the difference between the modelled seismicity of the scenarios may help to choose the optimal one in regards to seismic or ground motion hazard.

\section{Acknowledgement}

The work was done under IMS Patronage project 'Modelling and Assimilation of Seismicity using Boundary Element Method (A-MAS)' supported by El Teniente Mine, Chile; Anglo Gold Ashanti, South Africa; Sibanye Gold Venture, South Africa; and Harmony, South Africa.

Renison and Barrick, Hemlo Operations: Williams Mines kindly permitted to use their data. We are grateful to the senior geotechnical engineers of these mines - Kevin Stacey and Phil Earl - for providing the required information and for interesting discussion of the results.

We also thank anonymous reviewers for valuable comments.

\section{References}

Linkov, AM 2005, 'Numerical modeling of seismic and aseismic events in geomechanics', Journal of Mining Science, vol. 41, no. 1, pp. 14-26.

Linkov, AM 2013, 'Keynote lecture: Numerical modelling of seismicity: Theory and applications', in A Malovichko \& D Malovichko (eds), Proceedings of the 8th International Symposium on Rockbursts and Seismicity in Mines: RaSiM8, Geophysical Survey of Russian Academy of Sciences, Obninsk, Mining Institute of Ural Branch of Russian Academy of Sciences, Perm, pp. 197-218.

McKinnon, SD 2006, 'Triggering of seismicity remote from active mining excavations', Rock Mechanics and Rock Engineering, vol. 39 , no. 3, pp. 255-279.

Salamon, MDG 1993, 'Keynote address: Some applications of geomechanical modelling in rockburst and related research', in P Young (ed.), Proceedings of the 3rd International Symposium on Rockbursts and Seismicity in Mines, A.A. Balkema, Rotterdam, pp. 297-309.

Sellers, EJ \& Napier, JAL 2001, 'A point kernel representation of large-scale seismic activity in mining', in G van Aswegen, RJ Durrheim \& WD Ortlepp (eds), Proceedings of the 5th International Symposium on Rockbursts and Seismicity in Mines, South African Institute of Mining and Metallurgy, Johannesburg, pp. 405-411.

Spottiswoode, S 2001, 'Keynote address: Synthetic seismicity mimics observed seismicity in deep tabular mines', in G van Aswegen, RJ Durrheim \& WD Ortlepp (eds), Proceedings of the 5th International Symposium on Rockbursts and Seismicity in Mines, South African Institute of Mining and Metallurgy, Johannesburg, pp. 371-377. 